\title{
Remarks on Schur's conjecture
}

\author{
Filip Morić János Pach \\ Ecole Polytechnique Fédérale de Lausanne \\ $\{$ filip.moric, janos.pach\}@epfl.ch *
}

\begin{abstract}
Let $P$ be a set of $n$ points in $\mathbb{R}^{d}$. It was conjectured by Schur that the maximum number of $(d-1)$-dimensional regular simplices of edge length $\operatorname{diam}(P)$, whose every vertex belongs to $P$, is $n$. We prove this statement under the condition that any two of the simplices share at least $d-2$ vertices and we conjecture that this condition is always satisfied.
\end{abstract}

\section{Introduction}

The investigation of various properties of graphs of distances generated by a finite set of points in Euclidean space was initiated by Erdős in 1946, and it has become a classical topic in discrete and computational geometry, with applications in combinatorial number theory, the theory of geometric algorithms, pattern recognition, etc. A typical problem in the area is Erdös's unit distance problem [2, 10]: what is the maximum number of unit distance pairs among $n$ points in $\mathbb{R}^{d}$ ?

In the present paper, we concentrate on graphs of diameters. The diameter graph $D(P)$ of a finite set of points $P$ in $\mathbb{R}^{d}$ is the graph whose vertex set is $P$, and two vertices are connected by an edge if and only if their distance is the diameter of $P$.

Throughout this paper, $d$ will always denote an integer which is at least 2 .

One of the basic results concerning graphs of diameters was obtained by Hopf and Pannwitz in 1934 [4]: the maximum number of diameters among $n$ points in the plane is $n$. In 3 dimensions, a similar result was conjectured by Vázsonyi and proved by Grünbaum [5], Heppes [6], and Straszewicz [11]: the maximum number of diameters generated by $n$ points in $\mathbb{R}^{3}$ is $2 n-2$. In higher dimensions, the analogous problem turned out to have a different flavor: Lenz found some simple constructions with a quadratic number of diameters.

In [9], instead of counting the number of edges, Schur, Perles, Martini, and Kupitz initiated the investigation of the number of cliques in a graph of diameters. A $k$-clique, that is, a complete subgraph of $k$ vertices in the graph of diameters of $P$ corresponds to a regular $(k-1)$-dimensional simplex (or, in short, $(k-1)$-simplex) of side length $\operatorname{diam}(P)$ generated by $P$.

Theorem A (Schur et al.). Any finite subset $P \subset \mathbb{R}^{d}$ contains the vertices of at most one regular $d$-simplex of edge length $\operatorname{diam}(P)$.

The main result in [9] is the following.

${ }^{*}$ The authors gratefully acknowledge support from the Hungarian Science Foundation EuroGIGA Grant OTKA NN 102029, from the Swiss National Science Foundation Grant 200021-125287/1, and from the NSF Grant CCF-0830272. 
Theorem B (Schur et al.). Any set $P$ of $n$ points in $\mathbb{R}^{3}$ can generate at most $n$ equilateral triangles of side length $\operatorname{diam}(P)$.

Theorem B can be regarded as another 3-dimensional generalization of the Hopf-Pannwitz result, according to which any set of $n$ points in the plane has at most $n$ diameters. It was conjectured by Z. Schur (see [9]) that this result can be extended to all dimensions $d$.

Conjecture 1 (Schur). The number of d-cliques in a graph of diameters on $n$ points in $\mathbb{R}^{d}$ is at most $n$.

The fact that this bound is tight can be shown by a simple construction; see [9].

We can prove Schur's conjecture for point sets satisfying a special condition.

Theorem 2. The number of d-cliques in a graph of diameters on $n$ vertices in $\mathbb{R}^{d}$ is at most $n$, provided that any two d-cliques share at least $d-2$ vertices.

We do not have any example violating the additional condition and we believe that, in fact, it holds for all graphs of diameters. However, we were unable to prove that it is true in general.

Conjecture 3. Any two unit regular $(d-1)$-simplices in $\mathbb{R}^{d}$ must share at least $d-2$ vertices, provided the diameter of their union is one.

This is vacuously true for $d=2$. For $d=3$ it follows, e.g., from Dolnikov's theorem [1, 13] (a direct proof is given in [9]), and it is open for $d \geq 4$. We cannot even verify that two simplices must share at least one vertex (for $d \geq 4$ ), so this step would already be a breakthrough. We propose the following still weaker conjecture.

Conjecture 4. Given two unit regular $(d-1)$-simplices in $\mathbb{R}^{d}$ with $d \geq 3$, we can choose a vertex $u$ of one simplex and a vertex $v$ of the other one, so that $|u v| \geq 1$.

This is only known to be true for $d=3$. Obviously, Conjecture 3 , if true, would imply Conjecture 4. It seems that regularity of the simplices is not a crucial condition in Conjecture 4, and the following stronger version may be true.

Conjecture 5. Let $a_{1} \ldots a_{d}$ and $b_{1} \ldots b_{d}$ be two $(d-1)$-simplices in $\mathbb{R}^{d}$ with $d \geq 3$, such that all their edges have length at least $\alpha$. Then there exist $i, j \in\{1, \ldots, d\}$ such that $\left|a_{i} b_{j}\right| \geq \alpha$.

In other words, given $d$ red and $d$ blue points, we can find a red-blue distance that is at least as large as the smallest monochromatic distance. We can ask another more general question, which is probably very hard.

Problem 6. For given $d$, characterize all pairs $k, \ell$ of integers such that for any set of $k$ red and $\ell$ blue points we can choose a red point $r$ and a blue point $b$ such that $|r b|$ is at least as large as the smallest distance between two points of the same color.

From an easy packing argument one can see that there is a good choice of $r$ and $b$, whenever at least one of the numbers $k$ and $\ell$ is large enough. The following theorem is a first step towards Problem 6.

Theorem 7. For any set of $2 k$ points $a_{1}, \ldots, a_{k}, b_{1}, \ldots, b_{k}$ in $\mathbb{R}^{d}$ the following inequality holds:

$$
\max \left\{\left|a_{i} b_{j}\right|: 1 \leq i, j \leq k\right\} \geq \min \left\{\left|a_{i} a_{j}\right|,\left|b_{i} b_{j}\right|: 1 \leq i<j \leq k\right\},
$$

provided that $k \geq c \cdot \sqrt{d} \cdot 2^{\frac{3 d}{2}}$ with a large enough absolute constant $c$. 
Some generalizations of Theorems A and B to graphs of the $k$-th largest distances were established in [8. In this paper we show how these theorems can be extended to non-regular triangles in $\mathbb{R}^{3}$ whose all sides are large (i.e., among the $k$ largest distances). For a given finite set $P \subset \mathbb{R}^{3}$, we let $d_{1}>d_{2}>\ldots$ be all distinct inter-point distances generated by point pairs in $P$, so that by $d_{k}$ we denote the $k$-th largest distance generated by $P$.

Theorem 8. For any $k$ there is a constant $c_{k}$ such that the following holds: any set $P$ of $n$ points in $\mathbb{R}^{3}$ can generate at most $c_{k} n$ triangles whose all sides have length at least $d_{k}$.

This can be viewed as a 3-dimensional analogue of the well-known observation by Vesztergombi: the number of pairs at distance $d_{k}$ among $n$ points in the plane is at most $2 k n$ (see [14]). The analogous statement for large non-regular $(d-1)$-simplices in $\mathbb{R}^{d}$ probably holds for $d \geq 4$ as well, but this is open.

The corresponding result for non-regular tetrahedra in $\mathbb{R}^{3}$ is somewhat weaker in the sense that the bound depends not only on $k$, but also on the given tetrahedron. We will see in Section 4 that this kind of dependence is necessary.

Theorem 9. For any tetrahedron $T$ and any $k$ there is a constant $c(T, k)$ such that the following holds: any finite set $P$ of points in $\mathbb{R}^{3}$ spans at most $c(T, k)$ tetrahedra congruent to $T$, provided that all edges of $T$ have length at least $d_{k}$.

If Conjecture 5 holds, then Theorem 9 can be generalized to higher dimensions. As for the planar case, it is an easy exercise to show that, for every $k$, there is a constant $c_{k}$ such that any finite set of points in the plane spans at most $c_{k}$ triangles, whose all sides have length at least $d_{k}$.

\section{Proof of Theorem 2}

We start with two lemmas that are borrowed from [12], where they are attributed to [7].

Lemma 2.1 (Kupitz et al.). Let $a, b, c, d$ be points on a 2-sphere of radius at least $1 / \sqrt{2}$ such that $\operatorname{diam}\{a, b, c, d\}=1$ and $|a b|=|c d|=1$. Then the short great circle arcs ab and cd must intersect.

The maximum number of diameters in a finite set of points on a 2 -sphere is the same as in the plane, as long as the radius of the sphere is large enough, compared to the diameter of the set.

Lemma 2.2 (Kupitz et al.). Let $S^{2}$ be a sphere of radius at least $1 / \sqrt{2}$ in $\mathbb{R}^{3}$. If a set of $n$ points on $S^{2}$ has diameter 1 , then the diameter occurs at most $n$ times.

Next, we establish Theorem 2, which says that Schur's conjecture (Conjecture1) holds, provided that the given graph of diameters satisfies an additional condition: any two d-cliques share at least $d-2$ vertices.

Proof of Theorem 2. Assume without loss of generality that the diameter of our set is equal to 1. We can also assume that every vertex belongs to at least two $d$-cliques, since otherwise we can proceed by induction. We start with several geometric observations.

Note that the vertices of a $d$-clique represent $d$ affinely independent points, so their affine hull is $(d-1)$-dimensional, i.e., a hyperplane. Therefore, the affine hull of the $d$ vertices divides the space into two half-spaces. 
We will use the expression angle uvw and notation $\alpha(u, v, w)$ to refer to the following set of points:

$$
\alpha(u, v, w)=\left\{\mu_{1}(u-v)+\mu_{2}(w-v): \mu_{1}, \mu_{2} \geq 0\right\} .
$$

Lemma 2.3. If two $d$-cliques $a_{1} \ldots a_{d-2} x y$ and $a_{1} \ldots a_{d-2} z t$ share exactly $d-2$ vertices, then the open segment zt has exactly one common point with aff $\left(a_{1}, \ldots, a_{d-2}, x, y\right)$, which lies inside $\alpha(x, c, y)$, where $c=\frac{a_{1}+\cdots+a_{d-2}}{d-2}$ is the center of gravity of $a_{1} \ldots a_{d-2}$.

Proof. Since $\left|a_{i} x\right|=\left|a_{i} y\right|=\left|a_{i} z\right|=\left|a_{i} t\right|=1$ for all $i=1, \ldots, d-2$, and

$$
|c x|=|c y|=|c z|=|c t|=\sqrt{\frac{d-1}{2(d-2)}},
$$

we know that points $x, y, z, t$ lie on a 2 -sphere with center $c$ and radius $\geq 1 / \sqrt{2}$ (Figures $1(\mathrm{a})$, $1(\mathrm{~b})$ ) . Hence, we can apply Lemma 2.1 to points $x, y, z, t$ to conclude that the arcs $x y$ and $z t$ intersect at some point $p$. But then the segment $c p$ is contained in $\alpha(x, c, y)$ and it is intersected by the open segment $z t$. Therefore, the open segment $z t$ intersects aff $\left(a_{1}, \ldots, a_{d-2}, x, y\right)$ at a point which lies in $\alpha(x, c, y)$, and in no other point, since otherwise the two $d$-cliques would lie in the same hyperplane and would necessarily coincide by Theorem A.

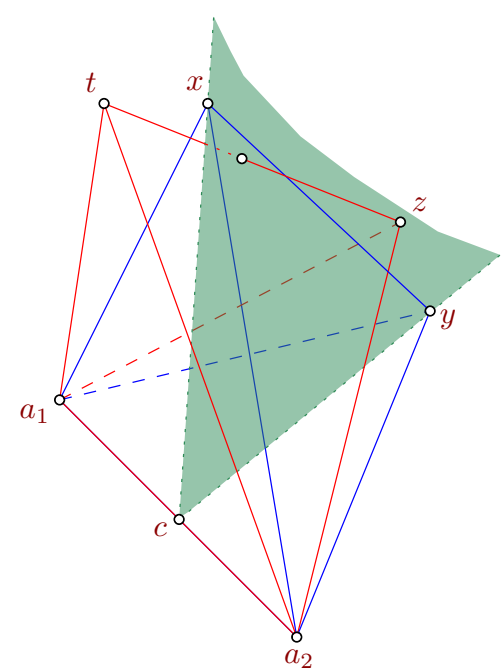

(a)

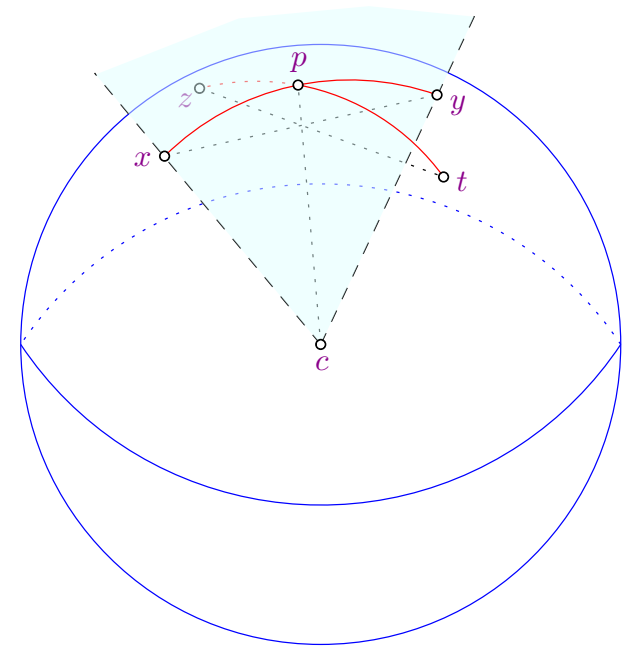

(b)

Figure 1: Proof of Theorem 2, Lemma 2.3

Lemma 2.4. There are no three $d$-cliques that share a $(d-1)$-clique.

Proof. Suppose the contrary: let $a_{1} \ldots a_{d-1} x, a_{1} \ldots a_{d-1} y$ and $a_{1} \ldots a_{d-1} z$ be three $d$-cliques. Denote by $c$ the center of gravity for $a_{1}, \ldots, a_{d-1}$. Then the points $x, y, z$ lie on the circle with center $c$ and radius $\sqrt{\frac{d}{2(d-1)}}$, that is orthogonal to aff $\left(a_{1}, \ldots, a_{d-1}\right)$. Since the radius of the circle is at least $1 / \sqrt{2}$, we have that $\angle x c y, \angle y c z, \angle z c x \leq \frac{\pi}{2}$. Hence, the points $x, y, z$ lie on a half-circle and we can assume without loss of generality that $y$ is between $x$ and $z$. Note that $|x y|,|y z|<1$ and 
points $x$ and $z$ lie on different sides of aff $\left(a_{1}, \ldots, a_{d-1}, y\right)$. According to our initial assumption, there is at least one $d$-clique $C$ containing $y$ apart from $a_{1} \ldots a_{d-1} y$. Since $C$ shares at least $d-2$ points with each of the cliques $a_{1} \ldots a_{d-1} x, a_{1} \ldots a_{d-1} y$ and $a_{1} \ldots a_{d-1} z$ and, moreover, $C$ cannot contain $x$ or $z$, we conclude that $C$ contains exactly $d-2$ of the points $a_{1}, \ldots, a_{d-1}$. Without loss of generality, let $C=y a_{1} \ldots a_{d-2} u$ and let $u$ lie on the same side of aff $\left(a_{1}, \ldots, a_{d-1}, y\right)$ as $x$. Now, because of Lemma 2.3, the open segment $a_{d-1} z$ contains a point from $\alpha\left(u, c^{\prime}, y\right)$, where $c^{\prime}$ is the center of gravity for $a_{1}, \ldots, a_{d-2}$. However, the whole set $\alpha\left(u, c^{\prime}, y\right)$ lies in the closed half-space that contains $x$, while the open segment $a_{d-1} z$ lies entirely in the open half-space that contains $z$. This is a contradiction.

It turns out that the above geometric observations provide enough information so that the proof can be finished more or less combinatorially.

Case 1. There is $a(d+1)$-clique $a_{1} \ldots a_{d+1}$.

Suppose there is a $d$-clique $C$ that contains a vertex $x \notin\left\{a_{1}, \ldots, a_{d+1}\right\}$. By the assumption, $C$ shares $d-2$ vertices with the clique $a_{1} \ldots a_{d}$, so we can assume that $C$ contains $a_{1}, \ldots, a_{d-2}$. But $C$ also shares $d-2$ vertices with the clique $a_{2} \ldots a_{d+1}$, so we can also assume that $C$ contains $a_{d-1}$. Therefore, $C=a_{1} \ldots a_{d-1} x$. Thus, we have three $d$-cliques containing $a_{1}, \ldots, a_{d-1}$ : namely, $a_{1} \ldots a_{d}, a_{1} \ldots a_{d-1} a_{d+1}$ and $C$. This is forbidden by Lemma 2.4. Thus, we conclude that all $d$-cliques must be contain in $a_{1} \ldots a_{d+1}$, which gives us at most $d+1$ cliques, so in this case the statement is proved, since $n \geq d+1$.

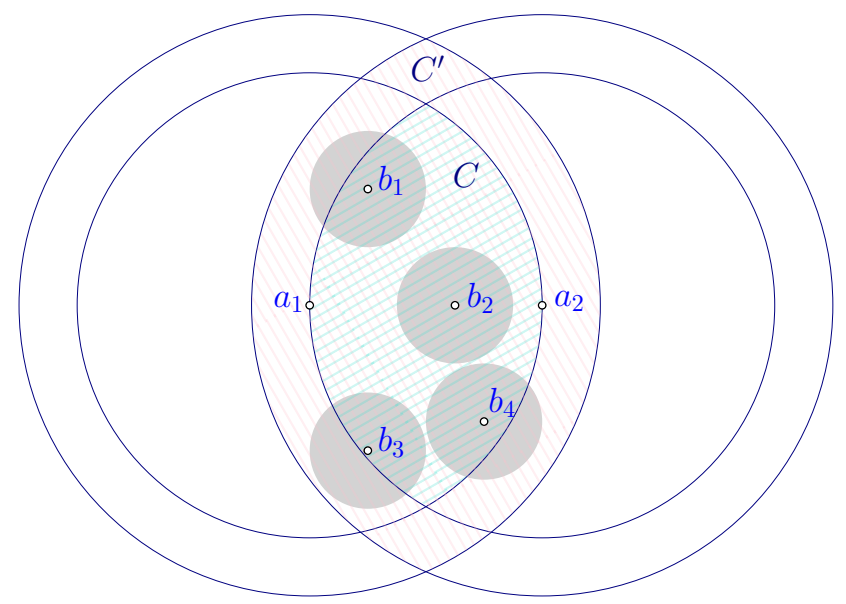

(a)

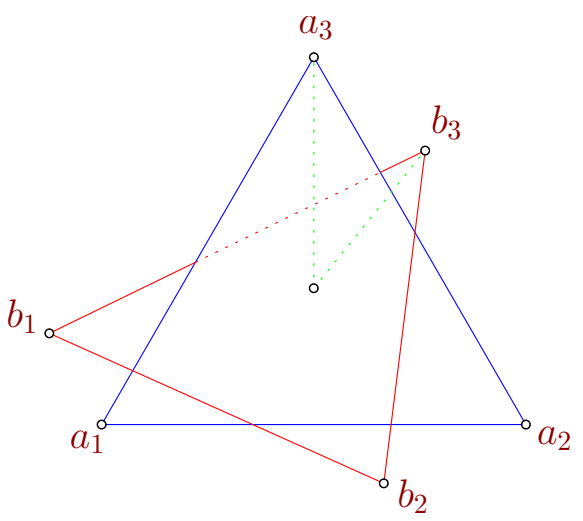

(b)

Figure 2: (a) Proof of Theorem 7) (b) construction for $d=4$ : two equilateral triangles in two orthogonal planes with a common center at the origin

Case 2. There is no $(d+1)$-clique.

We have several subcases.

Subcase 2.1 There are two d-cliques that share $d-1$ vertices.

Let the cliques be $a_{1} \ldots a_{d-1} x$ and $a_{1} \ldots a_{d-1} y$. Observe that $|x y|<1$, since we assume there is no $(d+1)$-clique. If there are no more $d$-cliques except for those generated 
by $a_{1}, \ldots, a_{d-1}, x, y$, we are done. So we can suppose that there are some more $d$-cliques. Any new $d$-clique shares $d-2$ points both with $a_{1} \ldots a_{d-1} x$ and with $a_{1} \ldots a_{d-1} y$. Hence, any new clique contains exactly $d-2$ of the vertices $a_{1}, \ldots, a_{d-1}$. We say that a $d$-clique is of type $k$ if it contains all the vertices $a_{1}, \ldots, a_{d-1}$ except for $a_{k}$. Now we will again branch out into different cases.

First, let us see what happens if all $d$-cliques have the same type, e.g., they all contain the points $a_{1}, \ldots, a_{d-2}$. The remaining two vertices of any $d$-clique must lie on the 2 sphere with center $\frac{a_{1}+\cdots+a_{d-2}}{d-2}$ and radius $\sqrt{\frac{d-1}{2(d-2)}}>\frac{1}{\sqrt{2}}$. Thus, the number of $d$-cliques is no more than the number of unit-diameters among $n-(d-2)$ points on a 2 -sphere of radius $>1 / \sqrt{2}$, which is at most $n-(d-2)$, by Lemma 2.2 .

Therefore, we can assume that there are at least two $d$-cliques of different types. Any two cliques of different types share exactly $d-3$ vertices among $a_{1}, \ldots, a_{d-1}$, so they must share at least one more vertex. Again, we consider different cases.

Suppose there are two $d$-cliques of different types that share a vertex $v$ outside of $\left\{a_{1}, \ldots, a_{d-1}, x, y\right\}$. Let the cliques be $a_{1} \ldots a_{d-2} u v$ and $a_{2} \ldots a_{d-1} v w$. Clearly, $a_{1} \ldots a_{d-1} v$ is also a $d$-clique, so we have three $d$-cliques sharing $d-1$ points $a_{1}, \ldots, a_{d-1}$, which is impossible, according to Lemma 2.4.

The second possibility that remains is that any two cliques of different types contain $x$ or $y$. This means that either all cliques (apart from the initial two) contain $x$ or all of them contain $y$. Without loss of generality, let all new cliques contain $x$. Notice that there can be at most one clique of each type, for if $C_{1}$ and $C_{2}$ were $d$-cliques of the same type, say, type 1 , there would be three $d$-cliques sharing $d-1$ points $x, a_{2}, \ldots, a_{d-1}$, contrary to Lemma 2.4. Consequently, in this case we have at most $d+1$ cliques, and the total number of vertices is at least $d+2$.

Subcase 2.2 Any two d-cliques share at most $d-2$ vertices.

Let $a_{1} \ldots a_{d-2} x y$ and $a_{1} \ldots a_{d-2} z t$ be two $d$-cliques. None of the points $x$ and $y$ forms a diameter with any of the points $z$ and $t$, since it would produce two $d$-cliques that share $d-1$ vertices. If all other cliques contain $a_{1}, \ldots, a_{d-2}$, we are done as above, so without loss of generality suppose that there is a $d$-clique $a_{1} \ldots a_{d-3} x u v$. Clearly, $u, v$ are new points, i.e., different from $a_{1}, \ldots, a_{d-2}, x, y, z, t$. But now $a_{1} \ldots a_{d-3} x u v$ and $a_{1} \ldots a_{d-2} z t$ have only $d-3$ points in common, contradicting the assumption.

We proved that $n$ is an upper bound for the number of $d$-cliques. A construction that this bound can be achieved was given in [9]. This completes the proof of Theorem 2 .

Remark. The weakest version of Conjecture 3 we can think of seems to be the following: There is a constant $K(d)$ such that among any $K(d)$ cliques in a graph of diameters, there are two cliques sharing a vertex. This would give a bound of the form $k(d) \cdot n$ for Schur's conjecture. However, it appears that even this weakest form requires a new insight.

It is natural to ask if there is a version of Conjecture 3 for cliques that might have fewer than $d$ vertices. In particular, is it true that a $d$-clique and a $(d-1)$-clique in a graph of diameters in $\mathbb{R}^{d}$ must share a vertex? For $d=2$ and $d=3$, this is clearly false. It is also false in $\mathbb{R}^{4}$, as shown by the following construction.

Proposition 2.1. There exist a unit regular tetrahedron and a unit regular triangle in $\mathbb{R}^{4}$ that do not share a vertex, while the diameter of their union is 1. 
Proof. Consider a unit regular tetrahedron abcd in $\mathbb{R}^{4}$ and let $u$ and $v$ be the midpoints of the edges $a b$ and $c d$. We have $|u v|=1 / \sqrt{2}$. Extend the segment $u v$ on both sides by an equal length to get a segment $x y$ of length 1 . Then the largest distance from $x$ and $y$ to a vertex of the tetrahedron is $\sqrt{\frac{5}{8}+\frac{1}{2 \sqrt{2}}}$. Let the origin coincide with the center of the tetrahedron and let the tetrahedron lie in $x_{4}=0$. Translate the points $x$ and $y$ by the vector $\left(0,0,0, \frac{\sqrt{3}}{2}-\sqrt{5 / 8}\right)$ to get points $p$ and $q$ and let $r=(0,0,0,-\sqrt{5 / 8})$. Now $p q r$ is an equilateral triangle of side 1 and all the distances among the points $p, q, r, a, b, c, d$ are at most 1 .

\section{Proof of Theorem 7}

Proof of Theorem 7 . Suppose the contrary, i.e., that the maximum is strictly smaller than the minimum. Without loss of generality, we assume that

$$
\min \left\{\left|a_{i} a_{j}\right|,\left|b_{i} b_{j}\right|: 1 \leq i<j \leq k\right\}=1
$$

and $\left|a_{1} a_{2}\right|=1$. Denote by $C$ the intersection of two balls with centers $a_{1}$ and $a_{2}$ and radius 1 (Figure 2(a)). Then $C$ contains all the points $b_{1}, \ldots, b_{k}$. Since $\left|b_{i} b_{j}\right| \geq 1$, the balls centered at $b_{1}, \ldots, b_{k}$ with radii $\frac{1}{2}$ do not overlap. Moreover, all these balls are contained in $C^{\prime}$, which is the intersection of the balls with centers $a_{1}$ and $a_{2}$ and radius $\frac{3}{2}$. Let us estimate the volume of $C^{\prime}$. Using the fact that the volume of a spherical cap of height $h$ is

$$
\frac{\pi^{\frac{d-1}{2}} r^{d}}{\Gamma\left(\frac{d+1}{2}\right)} \int_{0}^{\arccos \frac{r-h}{h}} \sin ^{d}(t) d t,
$$

where $r$ is the radius of the sphere, we get

$$
\begin{array}{r}
\operatorname{Vol}\left(C^{\prime}\right)=2 \cdot \frac{\pi^{\frac{d-1}{2}}(3 / 2)^{d}}{\Gamma\left(\frac{d+1}{2}\right)} \int_{0}^{\arccos \frac{1}{3}} \sin ^{d}(t) d t \\
\leq 2 \cdot \frac{\pi^{\frac{d-1}{2}(3 / 2)^{d}}}{\Gamma\left(\frac{d+1}{2}\right)} \cdot\left(\frac{2 \sqrt{2}}{3}\right)^{d} \cdot \arccos \frac{1}{3}=O\left(\frac{(2 \pi)^{\frac{d}{2}}}{\Gamma\left(\frac{d+1}{2}\right)}\right) .
\end{array}
$$

But $C^{\prime}$ contains $k$ non-overlapping balls of radius $\frac{1}{2}$, and, therefore,

$$
k \cdot \frac{\pi^{\frac{d}{2}} 2^{-d}}{\Gamma\left(1+\frac{d}{2}\right)} \leq O\left(\frac{(2 \pi)^{\frac{d}{2}}}{\Gamma\left(\frac{d+1}{2}\right)}\right) .
$$

Finally, taking into account the asymptotics $\Gamma(x) \sim x^{x-\frac{1}{2}} e^{-x} \sqrt{2 \pi}$, we obtain $k=O\left(\sqrt{d} \cdot 2^{3 d / 2}\right)$, with a contradiction.

Remark. On the other hand, we know that Theorem 7 does not hold with $k \leq\left\lceil\frac{d+1}{2}\right\rceil$. To see this, consider the following construction. Let $a_{1} \ldots a_{k}$ be a regular $(k-1)$-dimensional simplex inscribed in the sphere

$$
\left\{\left(x_{1}, \ldots, x_{d}\right): x_{1}^{2}+\cdots+x_{k-1}^{2}=1, x_{k}=\cdots=x_{d}=0\right\}
$$

and let $b_{1} \ldots b_{k}$ be a regular $(k-1)$-dimensional simplex inscribed in the sphere

$$
\left\{\left(x_{1}, \ldots, x_{d}\right): x_{k}^{2}+\cdots+x_{2 k-2}^{2}=1, x_{1}=\cdots=x_{k-1}=0\right\} .
$$


Then $\left|a_{i} a_{j}\right|=\left|b_{i} b_{j}\right|=\sqrt{\frac{2 k}{k-1}}$ for all $i \neq j$, while $\left|a_{i} b_{j}\right|=\sqrt{2}$ (Figure 2(b) .

Thus, the smallest $k(d)$ for which Theorem 7 holds is somewhere between $d / 2$ and $c \sqrt{d} \cdot 2^{\frac{3 d}{2}}$. The gap is obviously quite large, and Conjecture 5 suggests the answer should be closer to the lower bound.

\section{Proofs of Theorems 8 and 9}

The proofs of Theorem 8 and Theorem 9 are both analogous to the proofs of the corresponding statements for regular simplices given in [8], with the only new ingredient being the next lemma.

Lemma 4.1. Let $a_{1} a_{2} a_{3}$ and $b_{1} b_{2} b_{3}$ be two triangles in $\mathbb{R}^{3}$ such that all their sides have length at least $\alpha$. Then there exist $i, j \in\{1,2,3\}$ such that $\left|a_{i} b_{j}\right| \geq \alpha$.

Proof. Suppose the contrary, i.e., that the two triangles are placed so that $\left|a_{i} b_{j}\right|<\alpha$ for all $i$ and $j$. Without loss of generality, let $a_{1} a_{2} a_{3}$ lie in the plane $x_{3}=0$. By the pigeon hole there are two vertices of $b_{1} b_{2} b_{3}$ that lie on the same side of $x_{3}=0$. Without loss of generality, let $b_{1}$ and $b_{2}$ lie in the half-space $x_{3} \geq 0$ and let $b_{1}=(0,0, p)$ and $b_{2}=(0, q, r)$, where $p, q, r$ are non-negative and $r \geq p$ (Figure 4). Translate the points $b_{1}$ and $b_{2}$ by vector $(0,0,-p)$ to get new points $c_{1}=(0,0,0)$ and $c_{2}=(0, q, r-p)$. Note that $\left|c_{1} c_{2}\right|=\left|b_{1} b_{2}\right| \geq \alpha$ and $\left|c_{i} a_{j}\right| \leq\left|b_{i} a_{j}\right|<\alpha$ for all $i \in\{1,2\}$, $j \in\{1,2,3\}$. It follows that the points $a_{1}, a_{2}, a_{3}$ must have non-negative second coordinates. Now we rotate the point $c_{2}$ around $c_{1}$ in the plane $x_{1}=0$ until it hits the plane $x_{3}=0$. Thus, we replace $c_{2}$ by $c_{2}^{\prime}=(0, s, 0)$, where $s=\sqrt{q^{2}+(r-p)^{2}}$. Again, $\left|c_{1} c_{2}^{\prime}\right|=\left|c_{1} c_{2}\right| \geq \alpha$ and the distances between $c_{2}^{\prime}$ and $a_{j}$ for $j \in\{1,2,3\}$ are all smaller than $\alpha$. Indeed, letting $a_{j}=(t, u, 0)$, we have

$$
\left|c_{2}^{\prime} a_{j}\right|=\sqrt{t^{2}+(u-s)^{2}} \leq \sqrt{t^{2}+(q-u)^{2}+(r-p)^{2}}=\left|c_{2} a_{j}\right|<\alpha,
$$

where we used that $u \geq 0$ and $q \leq s$.

The points $c_{1}, c_{2}^{\prime}, a_{1}, a_{2}, a_{3}$ lie in the same plane and segment $c_{1} c_{2}^{\prime}$ can intersect at most two

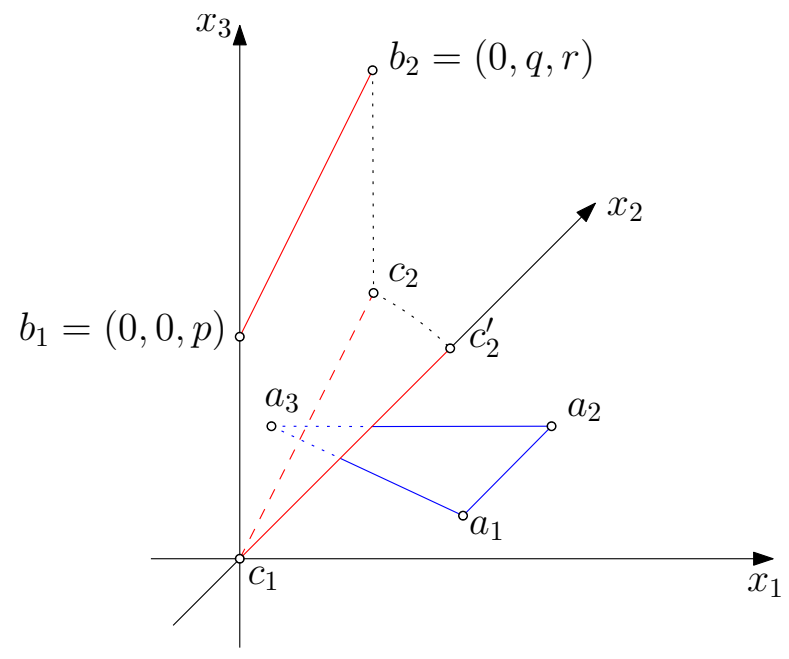

Figure 3: Proof of Lemma 4.1 
sides of triangle $a_{1} a_{2} a_{3}$ at their interior points. So, without loss of generality, assume that $c_{1} c_{2}^{\prime}$ does not intersect $a_{1} a_{2}$ at an interior point. Then either $c_{1}, c_{2}^{\prime}, a_{1}, a_{2}$ are in convex position or an extension of one of the segments $c_{1} c_{2}^{\prime}$ and $a_{1} a_{2}$ intersects the other one. In either case one can easily show that one of the segments $c_{1} a_{1}, c_{1} a_{2}, c_{2}^{\prime} a_{1}, c_{2}^{\prime} a_{2}$ has length at least $\min \left\{\left|c_{1} c_{2}^{\prime}\right|,\left|a_{1} a_{2}\right|\right\} \geq \alpha$. Contradiction.

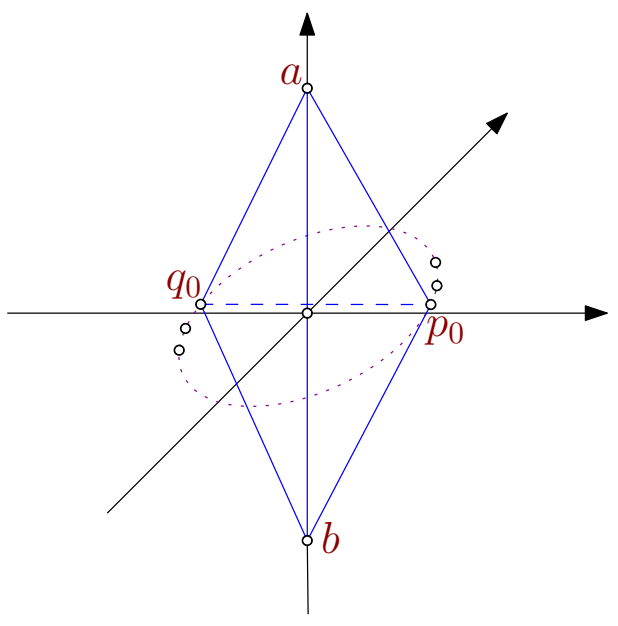

(a)

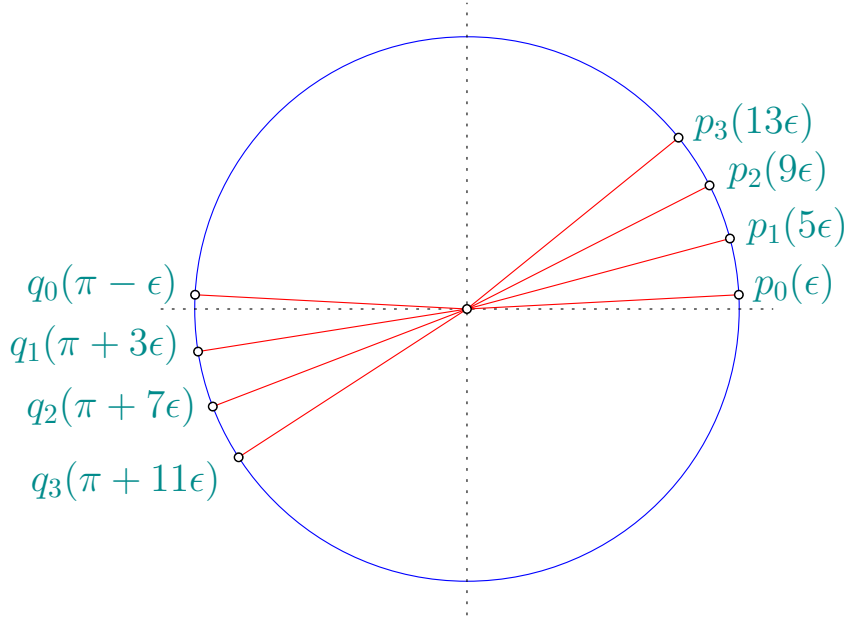

(b)

Figure 4: (a) Construction with many congruent large non-regular simplices; (b) points in the plane $x_{3}=0$

Remark. Note that dependence on $T$ is necessary in Theorem 9, as shown by this simple construction. Take two points $a=(0,0,1), b=(0,0,-1)$, and $2 n$ points in the plane $x_{3}=0$ on the circle $x_{1}^{2}+x_{2}^{2}=1 / 4$ with polar coordinates as follows:

$$
p_{i}=\left(\frac{1}{2},(1+4 i) \epsilon\right), \quad q_{i}=\left(\frac{1}{2}, \pi+(4 i-1) \epsilon\right)
$$

for $i=0,1, \ldots, n-1$ and small enough $\epsilon>0$ (Figures $4(\mathrm{a}) 4(\mathrm{~b})$. In this set of $2 n+2$ points we have that

$$
d_{1}=|a b|=2, d_{2}=\left|a p_{i}\right|=\frac{\sqrt{5}}{2} \text { and } d_{3}=\left|p_{i} q_{i}\right|=\sqrt{\frac{1}{2}+\frac{1}{2} \cos (2 \epsilon)}<1
$$

(recall that the distance between the points $\left(r_{1}, \theta_{1}\right)$ and $\left(r_{2}, \theta_{2}\right)$ in polar coordinates is equal $\left.\sqrt{r_{1}^{2}+r_{2}^{2}-2 r_{1} r_{2} \cos \left(\theta_{1}-\theta_{2}\right)}\right)$. Also, we can check that for all $i, j$ we have

$$
\left|p_{i} q_{j}\right|=\sqrt{\frac{1}{2}+\frac{1}{2} \cos ((4(j-i)-2) \epsilon)} \leq \sqrt{\frac{1}{2}+\frac{1}{2} \cos (2 \epsilon)}=d_{3},
$$

since $|4(j-i)-2| \geq 2$. It remains to notice that the chosen points span $2 n-1$ tetrahedra with edge lengths $d_{1}, d_{2}, d_{2}, d_{2}, d_{2}, d_{3}$. Those are the tetrahedra $a b p_{i} q_{j}$ for all $i, j \in\{0,1, \ldots, n-1\}$ such that $j-i \in\{0,1\}$. Thus, for $k=3$ we can have an arbitrarily large number of tetrahedra whose all edges have lengths at least $d_{k}$. 


\section{References}

[1] V. L. Dolnikov: Some properties of graphs of diameters, Discrete Comput. Geom. 24 (2000), 293-299.

[2] P. Erdős: On sets of distances of n points, Amer. Math. Monthly 53 (1946) 248-250.

[3] P. Erdős, J. Pach: Variations on the theme of repeated distances, Combinatorica 10 (1990), pp. 261-269

[4] H. Hopf, E. Pannwitz: Aufgabe Nr. 167, Jahresbericht Deutsch. Math.-Verein. 43 (1934) p. 114.

[5] B. Grünbaum: A proof of Vászonyi's conjecture, Bull. Res. Council Israel, Sect. A 6 (1956) $77-78$.

[6] A. Heppes: Beweis einer Vermutung von A. Vázsonyi, Acta Math. Acad. Sci. Hungar. 7 (1956) 463-466.

[7] Y. S. Kupitz, H. Martini and B. Wegner: Diameter graphs and full equi-intersectors in classical geometries, IV International Conference in "Stoch. Geo., Conv. Bodies, Emp. Meas. \& Apps. to Eng. Sci.", Vol. II, Rend. Circ. Mat. Palermo (2) Suppl. No. 70, part II (2002), 65-74.

[8] F. Morić, J. Pach: Large simplices determined by finite point sets, preprint.

[9] Z. Schur, M. A. Perles, H. Martini, Y. S. Kupitz: On the number of maximal regular simplices determined by $n$ points in $\mathbb{R}^{d}$, in: Discrete and Computational Geometry, The GoodmanPollack Festschrift, Aronov etc. eds., Springer, 2003, 767-787.

[10] J. Spencer, E. Szemerédi, W.T. Trotter: Unit distances in the Euclidean plane, in: Graph Theory and Combinatorics, B. Bollobás, ed., Academic Press, London, 1984, 293-303.

[11] S. Straszewicz: Sur un problème géométrique de P. Erdős, Bull. Acad. Pol. Sci., Cl. III 5 (1957) 39-40.

[12] K. J. Swanepoel: Unit distances and diameters in Euclidean spaces, Discrete \& Computational Geometry, 41 (2009), 1-27.

[13] K. J. Swanepoel: A new proof of Vázsonyi's conjecture, Journal of Combinatorial Theory, Series A, 115 (2008), 888-892.

[14] K. Vesztergombi: On large distances in planar sets, Discrete Math. 67 (1987) 191-198. 